\title{
Low-Temperature Heat Capasity Measurements in Gd-Based Nanocomposites
}

\author{
A. Berkutova ${ }^{a, *}$, A. Zelenakova ${ }^{a}, \mathrm{P} \cdot \mathrm{HrubovcaK}^{a, b}, \mathrm{O} \mathrm{Kapusta}^{a}$, \\ R. TARASEnKO ${ }^{a}$ AND V. ZELEnAK ${ }^{c}$ \\ ${ }^{a}$ Department of Condensed Matter Physics, University of P.J. Šafárik, Park Angelinum 9, 04001 Košice, Slovakia \\ ${ }^{b}$ Frank Laboratory of Neutron Physics, JINR, Dubna, Russia \\ ${ }^{c}$ Department of Inorganic Chemistry, University of P.J. Šafárik, Moyzesova 11, 04001 Košice, Slovakia
}

\begin{abstract}
The magnetocaloric effect (MCE) of hybrid nanostructure consisting of fine gadolinium oxide $\left(\mathrm{Gd}_{2} \mathrm{O}_{3}\right)$ nanoparticles with diameter up to $12 \mathrm{~nm}$ loaded into the pores of the periodically ordered mesoporous silica with cubic (SBA-16) symmetry were investigated. These studies were mainly based on heat capacity $C_{p}$ measurements in temperature range $0.4-55 \mathrm{~K}$ under external magnetic field from 0 to $9 \mathrm{~T}$. After the subtraction of lattice heat capacity contribution, the maximum of isothermal magnetic entropy change $\Delta S_{M}=33 \mathrm{~J} /(\mathrm{K} \mathrm{kg})$ and value of refrigerant capacity power RCP $\sim 170 \mathrm{~J} / \mathrm{kg}$ was obtained at temperature $2.5 \mathrm{~K}$. Our findings confirm that this kind of nanocomposites can be promising for future refrigeration technology applications.
\end{abstract}

DOI: 10.12693/APhysPolA.137.911

PACS/topics: magnetocaloric effect, $\mathrm{Gd}_{2} \mathrm{O}_{3}$ nanoparticles, nanoporous materials, magnetic entropy change, heat capacity

\section{Introduction}

For the last years there is a huge interest of using magnetic materials as essential parts in number of devises such as electromotors, permanent magnets, memory media, transducers, as well as in environmental and industrial technologies. In particular, researches in magnetic refrigeration (MR) are topic in many investigations nowadays. For instance, it is based on magnetocaloric effect (MCE) which is related to magneto-thermodynamic phenomenon when a temperature change is caused by the changing of external magnetic field [1].

E. Warburg was first who discovered MCE in iron (1881) [2]. In 1926-1927 P. Debye [3] and W. Giauque [4] each independently explained the process of reaching very low temperatures using method of adiabatic demagnetization. Many years later the low-temperature MCE was reported in a variety of paramagnetic salts such as ammonium iron (III) sulfate [5], chromium (III) potassium sulfate [6] and cerium magnesium nitrate [7] and successfully achieved temperatures below $1 \mathrm{~K}$ [8]. In the process of tailoring the qualities of potential refrigerants, magnetic nanoparticles have been found very perspective.

Despite of high attention focused on magnetocaloric materials design, there is relatively a little work devoted to the exploration of the properties of nanoparticle clusters or assemblies with respect to MCE. It is known that the origin of unique properties of nanoparticles is the result of size effects. In the case of ferromagnetic nanoparticles, after reducing the size of the particle

\footnotetext{
* corresponding author; e-mail: anna.berkutova@student.upjs.sk
}

below certain critical value, the particle remains in monodomain state. Hence the particle exhibits large magnetic moment, so-called superspin, which affects peculiar properties of the systems consisting of these particles. In comparison with bulk materials, nanoparticles are characterized by high surface to volume ratio, so it leads to an assumption that such structures would transfer heat with considerably higher efficiency. Establishing the relation between fundamental magnetization processes and magneto-thermal responses can help to improve and tune the applicability of magnetic nanoparticles.

V. Franco et al. [9] studied the field dependence of the magnetic entropy change peak at the low temperature surface spin freezing transition in chemically synthesized, monodispersed $\mathrm{Co}, \mathrm{Co}-\mathrm{Ag}$, and $\mathrm{Ni}-\mathrm{Ag}$ core-shell nanoparticles. They reported entropy change $\Delta S_{M} \sim 2.7 \mathrm{~J} /(\mathrm{K} \mathrm{kg})$. H. Zeng et al. [10] presented work in which magnetocaloric properties of the as-consolidated nanocrystalline and coarse-grained gadolinium metals were studied. It was shown that with decreasing $\mathrm{Gd}$ grains from micrometer to nanometer range, magnetic entropy change $\Delta S_{M}$ diminishes from 10.07 to $4.47 \mathrm{~J} /(\mathrm{K} \mathrm{kg})$, at a magnetic-field change of $5 \mathrm{~T}$ near room temperatures. R. Paul et al. [11] investigated $\mathrm{Gd}_{2} \mathrm{O}_{3}$ nanotubes in both parallel and perpendicular magnetic field orientations with respect to the axis of the $\mathrm{Gd}_{2} \mathrm{O}_{3}$ nanotube array and performed entropy change $\Delta S_{M \|} \sim 10 \mathrm{~J} /(\mathrm{K} \mathrm{kg})$ and $\Delta S_{M \perp} \sim 16 \mathrm{~J} /(\mathrm{K} \mathrm{kg})$.

At present, all efforts to design suitable magnetic refrigerator are focused on finding the alternative cooling technology used in regimes from the room to the cryogenic temperature. Particularly the magnetic refrigeration with its energy-efficient and environment-friendly advantages open the perspective of replacing currently used vapor-cycle refrigeration technology. 
To observe large magnetocaloric effect the compounds which contain atoms with many unpaired electrons are typically used. Each atom generates its own tiny magnetic moment and makes contribution to the refrigeration proses. Magnetic refrigerants commonly use the gadolinium ions $\left(\mathrm{Gd}^{3+}\right)$, because it has seven unpaired electrons what makes refrigeration more efficient.

Therefore, for this work we prepared Gd-based nanocomposite with $\mathrm{Gd}_{2} \mathrm{O}_{3}$ nanoparticles encapsulated into periodic nanoporous silica matrix with cubic symmetry. The aim was to study MCE by means of heat capacity $C_{p}$ measurements for future cryogenic magnetic refrigeration applications. To our knowledge, not much focus has been paid on the MCE in these materials.

\section{Experimental}

We investigated nanocomposite which was prepared by method of nanocasting $\mathrm{Gd}_{2} \mathrm{O}_{3}$ nanoparticles with diameter up to $12 \mathrm{~nm}$ into periodic nanoporous silica matrix. The structure of composite has been previously studied and published elsewhere [12]. For the mesoporous matrix SBA-16 preparation and modification were used following chemicals: $35 \% \mathrm{HCl}$ (Central chem.), 98\% TEOS tetraetoxysilane (Sigma Aldrich), Pluronic P-123 (Sigma Aldrich), Pluronic F-127 (Sigma Aldrich), distilled water, $99.9 \% 99.9 \% \mathrm{Gd}\left(\mathrm{NO}_{3}\right)_{3} \cdot 6 \mathrm{H}_{2} \mathrm{O}$. The mesoporous matrix SBA-16 with a cubic symmetry $(\operatorname{Im} 3 \mathrm{~m})$ was prepared by the procedure [13]. Prepared mesoporous matrix SBA-16 was modified employing the wet impregnation method by mixing $\mathrm{Gd}\left(\mathrm{NO}_{3}\right)_{3}$ solutions with porous matrix (SBA-16) by means of ultrasonication. The obtained solution was then centrifuged, separated and dried. Dried powders were calcinated.

To perform the temperature and magnetic field dependence of the heat capacity $C_{p}$ measurements we used the heat capacity option of commercial PPMS (Quantum Design) in temperature range $0.4-55 \mathrm{~K}$ and applied magnetic fields from 0 to $9 \mathrm{~T}$. The powder sample with the mass of $m=3 \mathrm{mg}$ was pressed to form a pellet and to make proper thermal contact between the pellet and a sample platform a very small amount of grease (Appiezon N) was used. Initial measurements of heat capacity $C_{p}$ were taken in the temperature interval $2-55 \mathrm{~K}$. Further, for more accurate studies, the temperature interval was supplemented with measurements at temperatures $0.4-2 \mathrm{~K}$ using Helium-3 Refrigerator Option of PPMS (Quantum Design).

\section{Results and discussion}

The microstructural characterization of studied sample was performed by high energy X-ray diffraction (HE-XRD) measurements using synchrotron radiation. XRD spectra document the presence of amorphous silica and gadolinium oxides of $\mathrm{Gd}_{2} \mathrm{O}_{3}$ type in the modified sample. The representative spectra of studied nanocomposites are presented in Fig. 1. Overlapping of

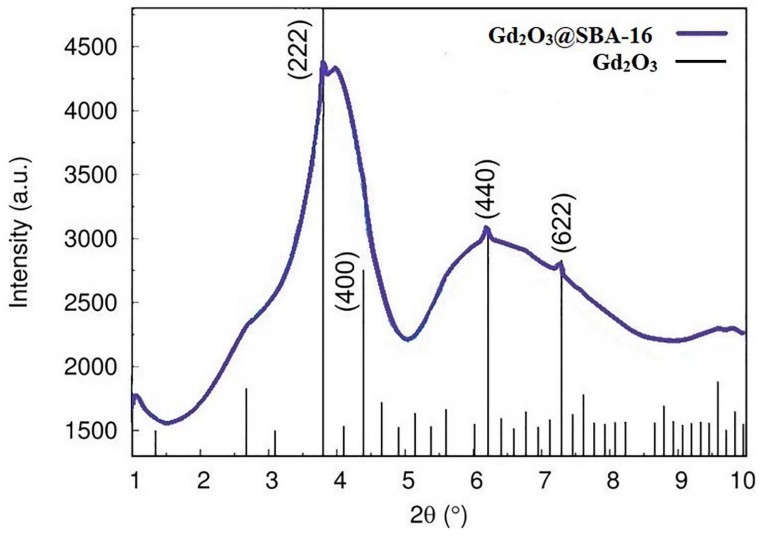

Fig. 1. Diffraction pattern of $\mathrm{Gd}_{2} \mathrm{O}_{3} @$ SBA-16 sample (purple line) with cubic matrix symmetry. The solid lines represent the peak positions of the gadolinium oxide reference (JCPDS No. 43-1014).

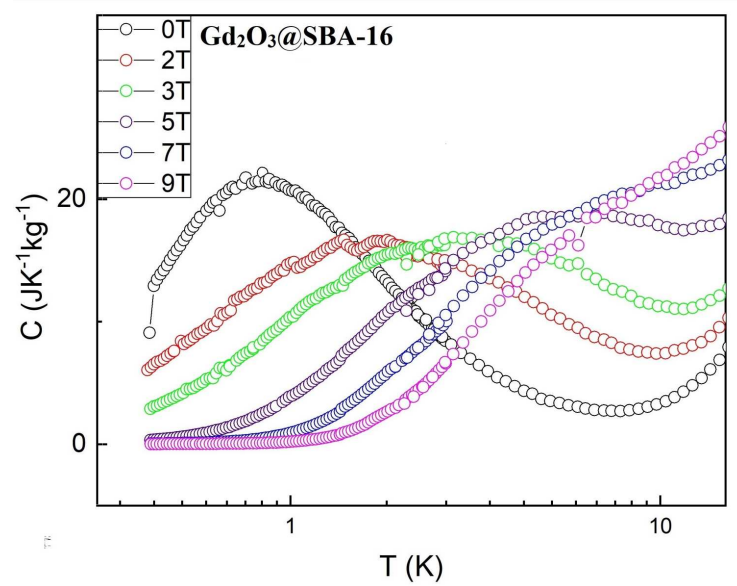

Fig. 2. The heat capacity of $\mathrm{Gd}_{2} \mathrm{O}_{3} @$ SBA-16 sample measured in a constant magnetic field values of $0,2,3$, 5,7 , and $9 \mathrm{~T}$.

diffraction peaks from amorphous silica and gadolinium oxide nanoparticles confirms the sufficient encapsulation of the NPs inside internal porous system.

The measurement of the heat capacity $C_{p}(H, T)$ as a function of temperature in constant magnetic fields and pressure provides the most complete characterization of MCE in magnetic materials. Figure 2 demonstrates the temperature dependence of heat capacity in our studied sample. The total heat capacity at constant pressure $C_{p}$ (tot) can be considered as a sum of magnetic contribution $C_{p}(\mathrm{mag})$ and lattice contribution $C_{p}$ (lat). This is represented by [14]

$$
\begin{gathered}
C_{p}(\text { tot })=C_{p}(\mathrm{mag})+C_{p}(\text { lat })= \\
\frac{a}{T^{2}}+b T^{3}+c T^{5}+d T^{7}+\ldots,
\end{gathered}
$$

where $a / T^{2}$ is magnetic contribution and remaining terms correspond to lattice contribution approximated within Debye model [15]. After subtracting the lattice 


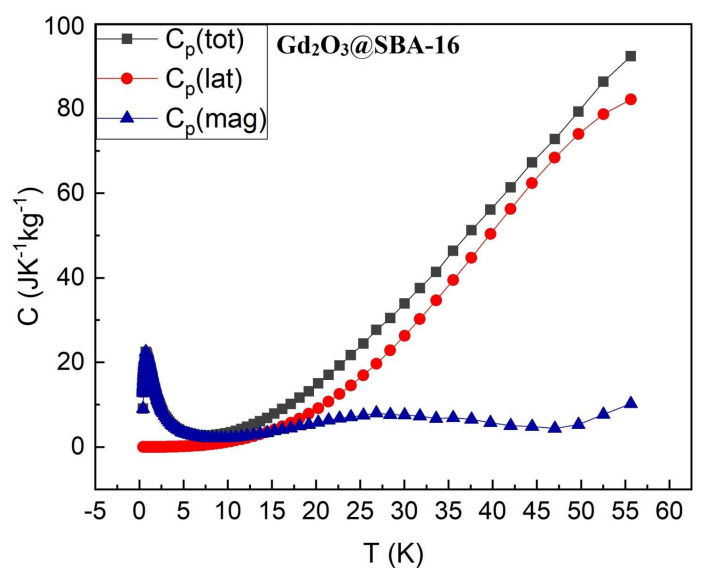

Fig. 3. Magnetic contribution of heat capacity $C_{p}$ (mag) was obtained after substracting the lattice contribution $C_{p}$ (lat) from total heat capacity $C_{p}$ (tot) measured in external magnetic field $0 \mathrm{~T}$.

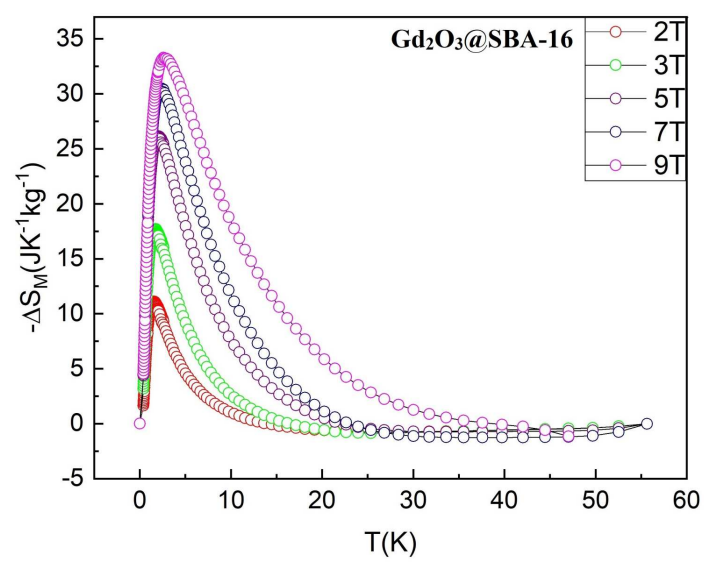

Fig. 4. The magnetocaloric effect of $\mathrm{Gd}_{2} \mathrm{O}_{3} @ \mathrm{SBA}-16$ sample as the magnetic entropy change $\Delta S_{M}$ calculated from the heat capacity data.

contribution from the experimentally determined heat capacity, the magnetic contribution was obtained, see Fig. 3.

The magnetocaloric response of the system represented by isothermal entropy change $\Delta S_{M}$ was calculated from heat capacity data using relation [16]

$$
-\Delta S_{M}(T, H)_{\Delta H}=\int_{0}^{T} \frac{C_{p}(T, H)-C_{p}(T, 0)}{T} \mathrm{~d} T .
$$

Temperature dependence of $-\Delta S_{M}$ at $\Delta H=2-9 \mathrm{~T}$ are shown in Fig. 4. When processing the data obtained in temperature interval $2-55 \mathrm{~K}$ by $\mathrm{He}^{4}$ cooling, it was noticed that considered temperatures are not suitable for proper analyses of such nanostructures. Further measurements were made at lower temperatures from 0.4 to $2 \mathrm{~K}$ by $\mathrm{He}^{3}$ cooling. Besides the maximum value of entropy change in studied nanocomposite $\Delta S_{M} \sim 33 \mathrm{~J} /(\mathrm{kg} \mathrm{K})$ is extremely high for this type of materials and established by method described in [16], values of RCP have been found RCP $\sim 170 \mathrm{~J} / \mathrm{kg}$, and Debye temperature $\theta_{\mathrm{D}}=57 \mathrm{~K}$ our heat capacity measurements confirmed mechanism of occurrence of MCE.

\section{Conclusions}

The entropy change and magnetocaloric response of $\mathrm{Gd}_{2} \mathrm{O}_{3} @$ SBA-16 nanocomposite prepared by nanocasting of of $\mathrm{Gd}_{2} \mathrm{O}_{3}$ nanoparticles into periodic nanoporous silica matrix with cubic symmetry were studied by heat capacity $C p$ measurements. The maximum value of entropy change in studied material at very low temperature $T \sim 2.5 \mathrm{~K}$, and the change in entropy with the change in applied magnetic field is reasonably large for this type of nanocomposites $\left(\Delta S_{M} \sim 33 \mathrm{~J} /(\mathrm{kg} \mathrm{K})\right)$. Along with the unique feature of nanoparticle systems and considering the high values of entropy change and lack of reports on investigation of similar nanoparticle systems, this kind of system appears to be promising candidate for the future cryogenic magnetic refrigeration applications.

\section{Acknowledgments}

This work was supported by the Development Operational Programme Research and Innovation for the project "New unconventional magnetic materials for applications", ITMS: 313011T544, co-funded by the European Regional Development Fund (ERDF).

\section{References}

[1] A.M. Tishin, Y.I. Spichkin, The Magnetocaloric Effect and its Applications CRC Press, Boca Raton 2003.

[2] E. Warburg, Ann. Phys. 13, 141 (1881).

[3] P. Debye, Ann. Phys. 81, 1154 (1926).

[4] W.F. Giauque, J. Am. Chem. Soc. 49, 1870 (1927).

[5] A.H. Cooke, Proc. Roy. Soc. A 62, 269 (1949).

[6] B. Bleaney, Proc. Roy. Soc. A 204, 216 (1950).

[7] A.H. Cooke, H.J. Duffus, W.P. Wolf, Philos. Mag. 44, 623 (1953).

[8] V.K. Pecharsky, K.A. Gschneidner Jr., J. Magn. Magn. Mater. 200, 44 (1999).

[9] V. Franco, A. Conde, D. Sidhaye, Appl. Phys. 107, 09A902 (2010).

[10] H. Zeng, J. Zhang, C. Kuang, Appl. Nanosci. 1, 51 (2011).

[11] R. Paul, T. Paramanik, K. Das, J. Magn. Magn. Mater. 417, 182 (2016).

[12] A. Zelenakova, O. Kapusta, V. Zelenak, Acta Phys. Pol. A 126, 218 (2014).

[13] T.W. Kim, R. Ryoo, M. Kruk, K.P. Gierszal, M. Jaroniec, S. Kamiya, O. Terasaki, J. Phys. Chem. $B$ 108, 11480 (2004).

[14] C.L. Snow, Q. Shi, J. Boerio-Goates, B.F. Woodfield, J. Phys. Chem. C 114 (49), 21100 (2010).

[15] N.K. Singh, K.G. Suresh, A.K. Nigam, S.K. Malik, A.A. Coelho, S. Gama, J. Magn. Magn. Mater. 317, 68 (2007).

[16] V. Franco, J.S. Blázquez, B Ingale, A. Conde, Annu. Rev. Mater. Res. 42, 305 (2012). 